\title{
Enfermedad celíaca del adulto
}

\section{Carolina Heredia Pla, Fabiola Castro P ${ }^{1 b}$, Joaquín Palma $\mathbf{H}^{\mathbf{1}}$. Adult celiac disease}

Celiac disease is a chronic enteropathy caused by intolerance to gluten. The true prevalence of this condition is much greater than previously recognized, with more silent cases being diagnosed and a greater proportion of cases diagnosed later in adulthood. It presents with a spectrum of clinical manifestations and histological abnormalities. Duodenal biopsy remains the gold standard for diagnosis of celiac disease, even though there are new serologic tests that are very specific. Correlation of clinical, serologic, and histological features are essential for a definitive diagnosis of celiac disease. The pathogenesis consists in an interaction between genetic, immunologic and environmental factors, which interact with HLA molecules to activate an immunologic response in the small bowel mucosa causing tissue damage. The main treatment is a free gluten diet. Health risks for untreated celiac disease appear to be greater compared with those patients who adhere to this treatment (Rev Méd Chile 2007; 135: 1186-94).

(Key words: Celiac disease; Gliadin; Gluten; Steatorrhea)
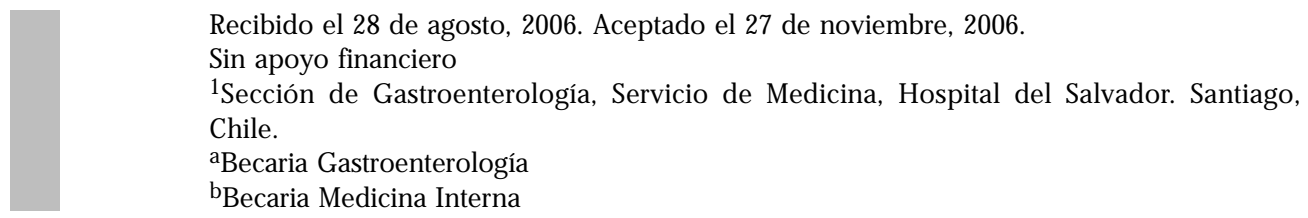

L a enfermedad celíaca (EC) se caracteriza por una inflamación crónica de la mucosa del intestino delgado por intolerancia al gluten, que resulta en atrofia vellositaria, malabsorción y síntomas clínicos que pueden manifestarse en la niñez y en el adulto ${ }^{1,2}$. La patogenia consiste en la interacción entre factores genéticos, inmunológicos y ambientales, existiendo marcadores genéticos específicos como HLA-DQ2 y HLA-DQ83. Las proteínas de la dieta presentes en el trigo, cebada, avena y centeno, interactúan con moléculas HLA para activar una respuesta inmunológica en la mucosa, produciendo daño tisular ${ }^{1}$.

Correspondencia a: Dra. Carolina Heredia P. Teléfono: 98849129. E mail: cherediap@gmail.com

La EC ha sido considerada hasta hace pocos años una enfermedad infrecuente, pero con el aumento de la sospecha clínica y el advenimiento de nuevos tests serológicos han permitido una mayor facilidad en el diagnóstico y aumento en su prevalencia.

\section{EPIDEMIOLOGÍA Y FACTORES DE RIESGO}

Clásicamente, la prevalencia de EC variaba desde 1:500 hasta 1:8.000 en la población general. Estudios recientes europeos, norteamericanos y sudamericanos sugieren una prevalencia entre $1: 150$ y $1: 300$, y que alrededor de $1 \%-3 \%$ de la población general de Europa y Estados Unidos de Norteamérica estará afectada en algún momento de la vida ${ }^{4-9}$. En Chile no existen estudios poblacionales que utilicen métodos serológicos. 
Mayor prevalencia de EC se encuentra en aquellos con predisposición familiar, y está asociada con enfermedades autoinmunes, como la artritis reumatoide, lupus eritematoso sistémico, enfermedad de Addison, enfermedad tiroidea autoinmune y hepatitis crónica activa. $\mathrm{Al}$ menos $7 \%$ de los pacientes con diabetes mellitus tipo I tienen también EC y aquellos con síndrome de Down, entre 5\%-12\%1,10.

No existe diferencia de $\mathrm{EC}$ entre hombres y mujeres ${ }^{12}$.

Entre los factores no genéticos asociados a la progresión de la EC se incluye la exposición a las gliadinas «n útero»o a través de la leche materna, dosis, edad de introducción e infecciones intestinales ${ }^{2}$.

\section{Modelo PATOgÉNICO EN LA ENFERMEDAD CELÍACA}

Un individuo susceptible a la enfermedad (con alelos DQ2 o DQ8) al ingerir gluten (presente en el trigo, cebada, centeno y avena), éste es digerido, quedando algunos péptidos ricos en prolina y glutamina que no fueron totalmente digeridos. Estos péptidos atraviesan la barrera epitelial intestinal para acceder a la región subepitelial. Se cree que la falla en la degradación de estas proteínas en el intestino delgado puede estar exagerada en individuos con EC activa que presentan importante daño epitelial ${ }^{3}$. Además, se ha especulado que la recaptación de péptidos derivados del gluten en la mucosa intestinal es facilitada por alguna infección o aumento en la permeabilidad de la mucosa, lo que ocurre a edades tempranas de la vida ${ }^{12}$. Estos datos son particularmente atractivos, pues pueden causar activación de células dendríticas, macrófagos y otras células inmunológicas en la lámina propia con un incremento en la producción de interferón y otras citoquinas en la mucosa del intestino delgado. Concomitantemente, el aumento en la liberación de transglutaminasa tisular durante la reparación de los tejidos asociados a infección, permitina que los péptidos ricos en prolina y glutamina que cruzaron la barrera epitelial, estén más capacitados para unirse a moléculas de clase II del complejo mayor de histocompatibilidad (DQ2 y DQ8) que se expresan en las células presentadoras de antígenos al convertir la glutamina en ácido glutámico. Por consecuencia, se presentan estos péptidos «extraños»a poblaciones de células T CD4 que los reconocen. Las células, en cambio, ponen en marcha mecanismos inflamatorios regulados por el balance entre citoquinas de perfil Th1 (interferón, factor de necrosis tumoral, interleuquinas 15 y 18) y además, activan la liberación de enzimas como metaloproteinasas de la matriz que pueden causar daño en la mucosa intestinal, con pérdida de la estructura vellosa e hiperplasia compensatoria de las criptas epiteliales $^{3,11,12}$.

\section{HISTORIA NATURAL}

El modelo tradicional de la enfermedad celíaca se muestra en la Figura 1, describiendo los distintos tipos de presentación: EC activa, silente y latente. En la EC activa se presenta la sintomatología

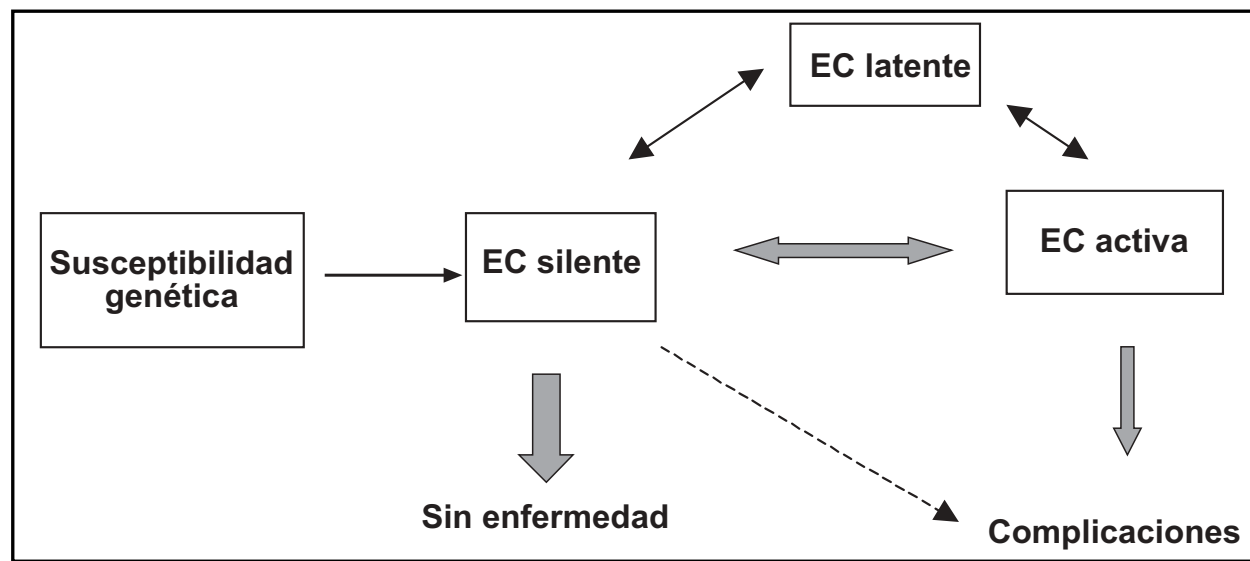

Figura 1. Historia natural de la enfermedad celíaca. 
clásica con malabsorción, diarrea crónica, esteatorrea o constipación, Sin embargo, existen casos sin malabsorción, incluso sólo con síntomas abdominales inespecíficos. La EC silente se caracteriza por la presencia de autoanticuerpos transglutaminasa IgA, lesiones histológicas típicas y genotipos específicos HLA-DQ en un individuo asintomático u oligosintomático con sintomatología atípica. La EC latente se caracteriza por individuos genéticamente susceptibles, pero sin manifestaciones clínicas ni histológicas. Pareciera que el tiempo, la calidad, y la carga de gluten, posiblemente combinado con otros desencadenantes ambientales o enfermedades intercurrentes, pueden ser determinantes en la edad de presentación en estos individuos. Algunos tienden a progresar y desarrollar una EC inequívoca, como también otros pueden revertir la enfermedad activa y volver a una etapa latente ${ }^{2,9,11,13}$.

\section{MANIFESTACIONES CLÍNICAS}

La inflamación en la EC afecta principalmente al intestino delgado proximal con variable compromiso del íleon distal. El intestino delgado tiene una considerable reserva funcional, lo que explica por qué algunos individuos presentan o no evidencia de malabsorción. La clínica es ampliamente variable y depende, entre otros factores, de la edad, sensibilidad al gluten y la cantidad de gluten ingerido en la dieta ${ }^{13}$.

Presentación clásica. Aparece en la infancia después de la introducción de cereales en la dieta, caracterizada por esteatomea, distensión abdominal, edema, palidez y letargia. La prevalencia de esta presentación ha variado con el desarmollo de métodos serológicos y sospecha clínica. En este sentido, la diamea ocurre en menos de $50 \%$ de los pacientes, comparado con casi $100 \%$ de aquellos que la presentaron en la década 1960-69. La baja de peso es actualmente poco común, incluso, al menos $30 \%$ de los pacientes tienen sobrepeso al diagnóstico $2,3,11,13$.

Presentación atípica. Los síntomas gastrointestinales pueden estar completamente ausentes o enmascarados por manifestaciones extraintestinales:

- Anemia por déficit de hierro: Es la causa más común de anemia, presentándose en 8\%-15\% de los individuos y pudiendo ser la única manifestación de la enfermedad. También la hemorragia gastrointestinal tiene un rol patogénico ${ }^{14,15}$. En un estudio con 17 pacientes, se detectó la presencia de sangrado gastrointestinal oculto en deposiciones en $25 \%$ de los adultos con EC con atrofia vellositaria parcial y en $54 \%$ de los pacientes con atrofia total $1,3,6,11,14-16$.

- Manifestaciones neurológicas: Ocurren en 10\% de los adultos con EC, principalmente ataxia cerebelosa (10\%-16\%), neuropatía periférica (49\%), epilepsia (3,5\%-5,5\%), demencia y depresión. Pocos estudios avalan el uso de inmunoglobulinas. La depresión y síntomas psiquiátricos son complicaciones comunes en aproximadamente un tercio de los pacientes. Factores como malabsorción, deficiencias nutricionales (principalmente déficit de vitamina B6) y la asociación con otras enfermedades autoinmunes pueden contribuir a la presencia de estos síntomas ${ }^{3,17}$.

- Osteopenia: Aparece por malabsorción de vitamina $\mathrm{D}$ y calcio, como también por la disminución de la ingesta de calcio por intolerancia a la lactosa. También contribuyen el género, la desnutrición e inactividad física. La osteopenia puede ser la única manifestación de la enfermedad en algunos casos. Responde a la dieta sin gluten con restauración gradual de hueso a los 2 años, por lo que el tratamiento debe ser precoz $3,14,15$.

- Manifestaciones cutáneas: Existe una asociación frecuente entre EC y dermatitis herpetiforme. La prevalencia es de alrededor de 1,2 por 100.000 personas y la prevalencia de HLA-DQ2 y DQ8 es la misma que para EC, apoyando el concepto que esta enfermedad es una manifestación de EC. Las lesiones pueden mejorar en semanas o meses con dieta libre de gluten asociado al uso de dapsona. Otras alteraciones cutáneas en EC son la psoriasis, estomatitis aftosa y alopecia $3,6,7,18$.

- Manifestaciones hepáticas: Se han asociado casos de EC y cirrosis biliar primaria y, en menor grado, con hepatitis crónica autoinmune y colangitis esclerosante primaria. En alrededor de $9 \%$ de los pacientes con EC se ha encontrado hipertransaminasemia idiopática que desaparece con la dieta sin gluten ${ }^{3,19}$.

- Diabetes Mellitus tipo 1: Es una de las condiciones asociadas a EC más conocidas. La 
prevalencia es de $1 \%$ a $16 \%$. Sobre $90 \%$ de los pacientes, la DM tipo 1 es diagnosticada antes de la EC, probablemente por la presencia de sintomatología más evidente y severa ${ }^{3,15}$.

\section{DiAgNóstico}

El diagnóstico se basa en la clínica, marcadores serológicos y biopsia de intestino delgado, la cual sigue siendo el patrón de oro por la mayoría de los investigadores ${ }^{20}$. La solicitud de estudios serológicos son recomendados en pacientes con: síntomas sugerentes de EC, condiciones asociadas con EC y familiares de primer grado de un paciente celíaco ${ }^{1}$.

El resultado positivo de los tests serológicos permite sospechar la EC, la cual debe confirmarse con biopsia de intestino delgado.

Biopsia. La histología se obtiene a través de una biopsia de la segunda porción del duodeno. Los

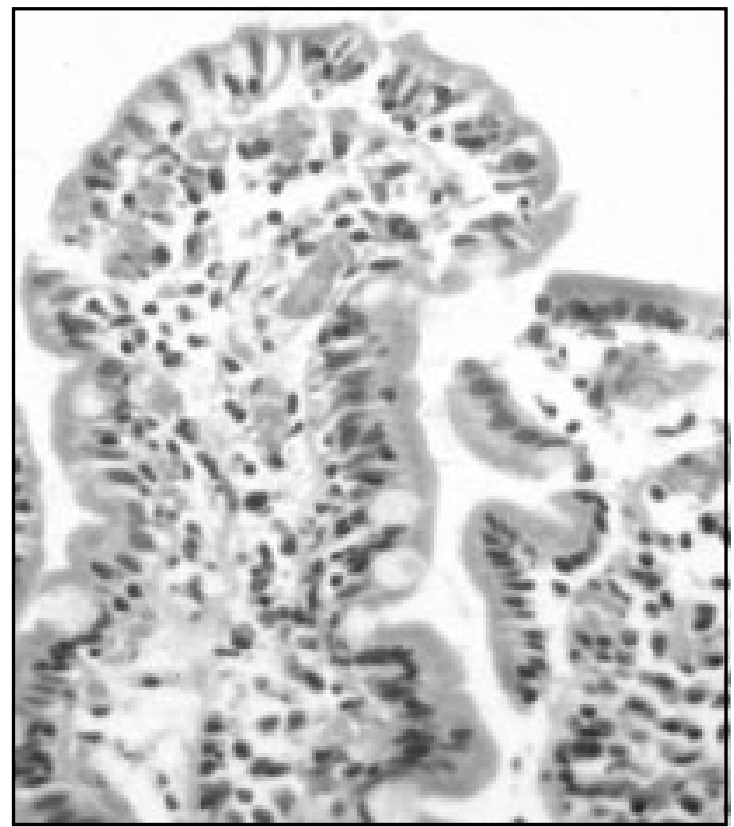

Figura 2. MARSH 1. Mucosa de intestino delgado con patrón vellositario y diferenciación epitelial adecuada (relación vellosidad/cripta $>2,5$ ) y aumento de linfocitos intraepiteliales ( $>40$ linfocitos/100 enterocitos). Lámina propia con leve infiltrado inflamatorio linfocitario y plasmocitario. hallazgos posibles de encontrar son vellosidades planas y atróficas, hiperplasia de criptas, pérdida de altura de los enterocitos e infiltrado linfocitario en la lámina propia.

La clasificación, o índice de Marsh, es utilizada para evaluar la progresión de la enfermedad en el tiempo ${ }^{21}$.

- Tipo I: Lesión infiltrativa: Caracterizada por un aumento de linfocitos intraepiteliales (superior a 30-40 linfocitos/100 células epiteliales), principalmente en el extremo distal de las vellosidades (Figura 2).

- Tipo II: Lesión hiperplásica: Aumento de linfocitos intraepiteliales asociado a una elongación de criptas y a un aumento de la actividad mitótica. Existe una disminución del índice vellosidad/cripta, que normalmente es superior a 3-5.

- Tipo III: Lesión destructiva: Atrofia vellositaria, ya sea parcial, subtotal o total (Figura 3).

- Tipo IV: Lesión hipoplásica: Atrofia total asociada a hipoplasia de criptas. Esta apariencia se asocia a EC refractaria y al desarrollo de linfoma de células $\mathrm{T}$ asociado a enteropatía.

Marcadores serológicos. En la práctica clínica pueden ser utilizados como métodos de búsqueda, apoyo diagnóstico o como monitorización y respuesta a una dieta sin gluten. Actualmente las

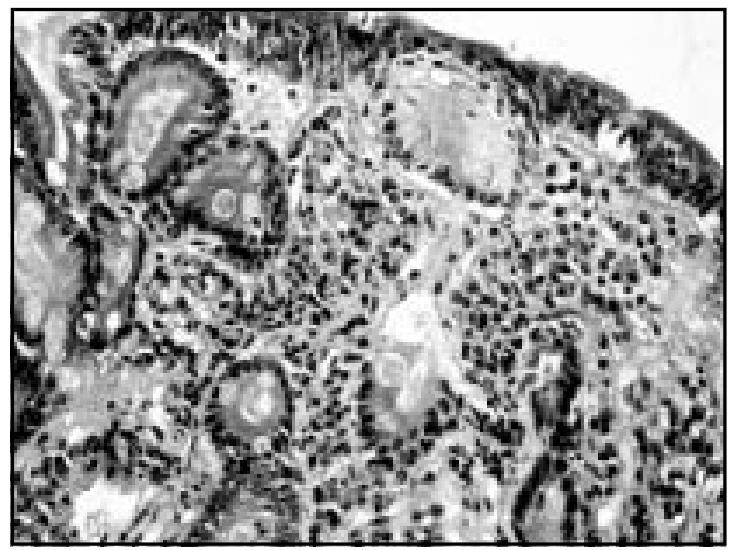

Figura 3. MARSH 3. Mucosa de intestino delgado con atrofia vellositaria difusa, elongación de las criptas (relación vellosidad/cripta $<0,5$ ) y aumento de linfocitos intraepiteliales ( $>40$ linfocitos/ 100 enterocitos). Lámina propia con moderado infiltrado inflamatorio linfocitario y plasmocitario. 
pruebas serológicas mejor aceptadas son los anticuerpos antiendomisio y los anticuerpos antitransglutaminasa tisular, no siendo necesario la medición de los anticuerpos antigliadina por su menor sensibilidad y especificidad ${ }^{1}$.

1. Anticuerpos antiendomisio (EMA). Estos anticuerpos están dirigidos contra una proteína (endomisio) del tejido conectivo ubicada entre la miofibrilla del músculo liso del tracto gastrointestinal.

EMA-IgA es el anticuerpo con mayor exactitud diagnóstica. Sensibilidad entre 97\% y 100\%, y especificidad entre 98\% y 99\%. Así, la combinación de AGAs y EMA tienen un valor predictivo positivo y negativo cercano a $100 \% 24-26$.

EMA-IgA se negativizan al excluir el gluten de la dieta, sin embargo tardan más tiempo en normalizarse que los AGA.

2. Anticuerpos antitransglutaminasa tisular (tTG). Estos anticuerpos se dirigen contra la enzima transglutaminasa (tTG), estableciéndose dos tipos de anticuerpos, IgA e IgG anti-tTG, ambos con alta sensibilidad entre $90 \%$ y $98 \%$ y especificidad entre $95 \%$ y $99 \%$, por lo que la asociación entre tTG -IgA y EMA-IgA aporta el mismo beneficio que cada uno por sí solo, dado que ambos poseen alta exactitud diagnóstica ${ }^{27}$.

Actualmente estos anticuerpos son muy útiles en el diagnóstico, pero por su alto costo limitan su uso. Para el seguimiento también son utilizados, normalizándose sus valores luego de 12 meses desde el tratamiento con dieta libre de gluten ${ }^{28}$.

3. Anticuerpos antigliadina (AGA). Existen dos tipos: Los de clase IgG tienen una sensibilidad entre $75 \%$ y $85 \%$ y una especificidad $75 \%$ y $90 \%$, con porcentaje de falsos positivos entre $30 \%$ y $50 \%$, siendo encontrados, por ejemplo, en enfermedad de Crohn, enteritis eosinofílica y en algunos individuos aparentemente sanos, por lo que su utilidad en la práctica es limitada 22

Los AGA de clase IgA tienen una sensibilidad 80\%-90\% y una especificidad entre 85\%-95\%. En caso de existir un déficit de IgA, los anticuerpos serán negativos (2\%) pudiendo existir falsos positivos en situaciones como la intolerancia a la leche y nefropatía por IgA. AGA-IgA tienen utilidad en la monitorización de adherencia y respuesta al tratamiento ${ }^{23}$.
En la práctica clínica, bastaría con solicitar Ac antitransglutaminasa para apoyo diagnóstico, pero en caso de no disponer de este test, se podría realizar la medición de Ac antiendomisio, al igual que para el seguimiento de los pacientes.

\section{TRATAMIENTO}

El manejo de la enfermedad celíaca se basa principalmente en la educación de la enfermedad, adherencia al tratamiento y corrección de deficiencias nutricionales mediante la incorporación de nutricionistas y gastroenterólogos. Una manera de recordar estos pilares se presenta en la Tabla 1.

Consejo dietético. El pilar fundamental del tratamiento es una dieta sin gluten.

Las principales fuentes de gluten son el trigo, la cebada y el centeno y todos los productos que contengan estos cereales deben ser evitados en todo paciente con EC, aun en aquellos asintomáticos, ya que éstos pueden tener carencia de micronutrientes que se acentúan incluso con pequeñas cantidades de gluten. Por otro lado, varios estudios han demostrado un aumento en el riesgo de mortalidad, de malignidad y de desórdenes inmunes en pacientes con EC que no se adhieren a un régimen estricto sin gluten ${ }^{28-32}$.

La inclusión de avena en la dieta es controvertida, ya que generalmente presenta contaminación con otros granos. Es recomendable un consumo promedio entre 50 y $60 \mathrm{~g}$ al día en casos leves o en aquellos pacientes en remisión, con control clínico y serológico para evitar una reactivación ${ }^{33-36}$.

Es importante tener presente que algunos pacientes presentan además patologías asociadas incluso temporales, como intolerancia a productos

\section{Tabla 1.}
C onsulta a un nutricionista
E ducación sobre la enfermedad
$\mathbf{L}$ arga adherencia a una dieta sin gluten
I dentificación y tratamiento de deficiencias nutricionales
A cceso a un grupo de trabajo
C ontinuo seguimiento por un grupo de expertos 
lácteos, a los huevos, a la soya y al arroz. Por el riesgo de graves carencias nutricionales, es fundamental evaluar el estado nutricional completo ${ }^{37}$.

Existe asociación entre EC e hipoesplenismo, por lo que es aconsejable la vacunación periódica antipneumocócica ${ }^{38}$.

Monitorización de la respuesta a una dieta sin gluten. La respuesta clínica e histológica es variable, pero en general los síntomas mejoran a las 2 semanas en $70 \%$ de los pacientes, y las lesiones histológicas a los 6 meses. El rol de la biopsia para seguimiento es discutido, ya que la serología permite identificar mejoría y adherencia ${ }^{38-40}$.

No respondedores. La causa más frecuente es la mala adherencia a la dieta o la ingestión inadvertida de gluten. Una vez descartadas estas causas, se pueden plantear 3 posibilidades:

- Enfermedades con clínica e histología similar tales como intolerancia a la lactosa, síndrome de intestino irritable, síndrome de sobrecrecimento bacteriano, enfermedad inflamatoria intestinal, colitis linfocítica o colágena, otros desórdenes.

- Enfermedad celíaca refractaria (ECR).

- Yeyunitis ulcerativa o linfoma intestinal. Estas entidades deben ser sospechadas en pacientes con ECR que no responden a corticoides.

Enfermedad celíaca refractaria (ECR). Se denomina ECR a una falla en la restauración estructural y funcional de la mucosa del intestino delgado a pesar de una dieta estricta sin gluten. Puede ser primaria, es decir, desde un inicio existe resistencia, o secundaria, donde inicialmente eran respondedores y se hacen refractarios ${ }^{41,42}$.

El diagnóstico es de exclusión y se asocia a una alta tasa de mortalidad.

$\mathrm{Su}$ patogénesis no ha sido aclarada pero algunos estudios han demostrado la existencia de anticuerpos antienterocitos en más de la mitad de los pacientes.

Luego de excluir las causas en estos casos, el tratamiento de ECR consiste en esteroides con buena respuesta en la mayoría de los casos. Sin embargo, la terapia no está exenta de riesgos ya que puede enmascarar la presencia de un linfoma ${ }^{43,44}$.

Han sido utilizados azatioprina y ciclosporina tanto para disminuir las dosis sistémicas de esteroi- des como para aquellos casos con refractariedad a la terapia esteroidal ${ }^{45-48}$. Existen algunas publicaciones con el uso de anticuerpos anti TNF (Infliximab) con buenos resultados en pacientes con falla de respuesta a otras terapias inmunomoduladoras, sin embargo, su uso no ha sido aprobado ampliamente ${ }^{49}$.

\section{ENFERMEDAD CELÍACA Y RIESGO DE CÁNCER}

Existe asociación entre EC y diversos tipos de carcinomas particularmente adenocarcinoma de intestino delgado, linfomas y carcinoma escamoso de boca y faringe ${ }^{50}$.

Linfoma no Hodgkin de células T asociada a enteropatía (EATL). Una vez diagnosticada la enfermedad celíaca, el linfoma puede tardar 5 a 10 años en desarrollarse y ocasionalmente la EC es detectada posterior al diagnóstico de linfoma. El EATL comúnmente se desarrolla en el yeyuno, pero también puede ser encontrado en íleon, linfonodos, estómago y colon. Frecuentemente es multifocal y está diseminado al momento del diagnóstico. La sospecha diagnóstica está dada por la aparición de baja de peso, diarrea persistente pese a la mantención de una dieta sin gluten, anorexia y dolor abdominal. Además es posible encontrar linfoadenopatías, hepatoesplenomegalia, masa abdominal o la aparición de un eritema cutáneo.

El pronóstico es pobre, aunque algunos pueden mejorar con quimioterapia y cirugía. En un reciente estudio de pacientes con EATL, la sobrevida a 30 meses fue sólo de 13\%51,52.

Adenocarcinoma de intestino delgado. Múltiples trabajos han documentado la asociación entre EC y carcinoma de intestino delgado. Un estudio del Reino Unido encontró una asociación de 13\%, con un tiempo medio de aparición de 8,2 años ${ }^{53}$.

La mayoría de los adenocarcinomas se encuentran en el yeyuno y duodeno, y su pronóstico es mejor que EATL con una sobrevida a 30 meses de $58 \%$, lo que se debe, en gran parte, a la posibilidad de resección quirúrgica completa.

Otros cánceres. Recientemente se ha confirmado asociación entre EC y cáncer orofaríngeo y esofágico con una tasa de incidencia estandarizada de 2,3 y de 4,2 , respectivamente ${ }^{54}$. 
Al parecer, existiría un aumento del riesgo de melanoma y cáncer de colon en pacientes con EC, sin embargo no existen mayores estudios que lo avalen ${ }^{54-56}$.

Por otra parte, el riesgo de cáncer de mama, y posiblemente de pulmón, parece estar disminuido en pacientes celíacos por razones no del todo aclaradas $54,57,58$.

\section{CONCLUSIONES}

La EC es una patología que requiere un alto índice de sospecha clínica, principalmente en aquellos países con gran consumo de cereales en la dieta.

En el adulto se puede presentar con síntomas inespecíficos o incompletos, incluso sólo con

\section{REFERENCIAS}

1. Elson CH, Bauew M, Barnard J, Bernstein S, Check I, CoHEN M ET AL. National Institutes of Health Consensus Development. Conference Statement on Celiac Disease, June 28-30, 2004. Gastroenterology 2005; 128: S1-S9.

2. Rewers M, Liu E, Simmons J, Redondo M, Hoffenberg E. Celiac disease associated with type 1 diabetes mellitus. Endocrinol Metab Clin N Am 2004; 33: 197-214.

3. Shamir R. Advances in celiac disease. Gastroenterol Clin N Am 2003; 32: 931-47.

4. Fasano A, CatASSI C. Current approaches to diagnosis and treatment of celiac disease: an evolving spectrum. Gastroenterology 2001; 120: 636-51.

5. Not T, Horvath K, HiL ID. Celiac disease risk in the USA: high prevalence of antiendomysium antibodies in healthy blood donors. Scand J Gastroenterol 1998; 33: 494-8.

6. Hovdenak N, Hovid E, Aksnes L. High prevalence of asymptomatic celiac disease in Norway: a study of blood donors. Eur J Gastroenterol Hepatol 1999; 11: 185-

7. Gandolfi L, Pratesi R, Cordoba JC. Prevalence of celiac disease in healthy blood donors in Brazil. Am J Gastroenterol 2000; 95: 689-92.

8. Trevisiol C, Not T, Berty I. Screening of celiac disease in healthy blood donors at two immuno- anemia ferropénica. El diagnóstico se debe realizar idealmente con EMA y tTG, siempre con confirmación histológica. El tratamiento se basa en una dieta libre de gluten que debe ser monitorizada en forma clínica y serológica. En caso de falla en la respuesta considerar EC refractaria y excluir la presencia de patología maligna por su alta mortalidad. En nuestro país, no existen datos epidemiológicos de la enfermedad, por lo que es necesario plantear una propuesta de fácil acceso para la detección e implementar una forma que aumente la educación y adherencia al tratamiento.

Agradecimientos: Al Dr. Salvador Navarro, Servicio de Gastroenterología, Hospital Clìnic i Provincial de Barcelona, España. transfusion centres in north-east Italy. Ital J Gastroenterol Hepatol 1999; 31: 584-6.

9. REWERS M. Epidemiology of Celiac Disease: What are the prevalence, incidente, and progression of Celiac Disease? Gastroenterology 2005; 128: S47-S51.

10. NeLSEN D. Gluten-Sensitive Enteropathy (Celiac Disease): More common than you think. Am Fam Physician 2002: 66: 2259-66, 2269-70.

11. American Gastroenterological Association. aGa Technical Review on Celiac Disease. Gastroenterology 2001; 120: 1526-40.

12. Kagnoff M. Overview and Pathogenesis of Celiac Disease. Gastroenterology 2005; 128: S10-S18.

13. Dejar D, Cicltira P. Clinical Features and Diagnosis of Celiac Disease. Gastroenterology 2005; 128: S19-S24.

14. Green P. The Many Faces of Celiac Disease: Clinical Presentation of Celiac Disease in the Adult Population. Gastroenterology 2005; 128: S74-S78.

15. MurRIA J. Celiac Disease in Patients with an Affected Member, Type 1 Diabetes, Iron Deficiency, or Osteoporosis? Gastroenterology 2005; 128: S52-S56.

16. FINE K. The prevalence of occult gastrointestinal bleeding in Celiac Sprue. N Engl J Med 1996; 334: 1163-7.

17. Bushara K. Neurologic Presentation of Celiac Disease. Gastroenterology 2005; 128: S92-S97. 
18. Zone J. Skin Manifestations of Celiac Disease. Gastroenterology 2005; 128: S87-S91.

19. Smyth C, Keuleher K, Keeling P. Hepatic manifestations of gastrointestinal diseases: Inflammatory bowel disease, celiac disease, and Whipple's disease. Clin Liver Dis 2002; 6: 1013-32.

20. FARREL R, KeLLY C. Diagnosis of celiac sprue. Am J Gastroenterol 2001; 96: 3237-4.

21. MARSH MN. Gluten, major histocompatibility complex, and the small intestine. A molecular and immunobiological approach to the spectrum of gluten sensitivity. Gastroenterology 1992; 102: 330-54.

22. Ribes M, Fotoulaki L, Biesa P, Augoustidou-Savopoulou M, Hernández F, Kanakoudi-Tsakalddes A Et AL. Celiac disease diagnosis: Similar high sensitivity for IgA-AGA using a home-made ELISA method in 2 European Pediatric Gastroenterology Units (PGU'S). Gut 1997; 41 (Suppl 3).

23. Kilander AF, Nilsson LA, Giliberg R. Serum antibodies to gliadin in celiac disease after gluten withdrawal. Scand J Gastroenterol 1987; 22: 29.

24. Uibo O, Uibo R, Kieimola V. Serum IgA anti-gliadin antibodies in an adult population sample. High prevalence without celiac disease. Dig Dis Sci 1993; 38: 2034.

25. Grodzinsky E, Hed J, Lueden G. Presence of IgA and IgG antigliadin antibodies in healthy adults as measured by micro-ELISA. Effect of various cutoff levels on specificity and sensitivity when diagnosing celiac disease. Int Arch Allergy Appl Immunol 1990; 92: 119.

26. Hed J, Leden G, Ottosson E. IgA anti-gliadin antibodies and jejunal mucosal lesions in healthy blood donors (letter). Lancet 1986; 2: 215.

27. Dieterich W, Ehnis T, Bauer M. Identification of tissue transglutaminase as the autoantigen of celiac disease. Nat Med 1997; 3: 797-801.

28. Hiш H. What Are the Sensitivity and Specificity of Serologic Tests for Celiac Disease? Do Sensitivity and Specificity Vary in Different Populations? Gastroenterology 2005; 128: S25-S32.

29. Ventura A, Magazzu G, Greco L. Duration of exposure to gluten and risk for autoimmune disorders in patients with celiac disease. Gastroenterology 1999; 117: 297.

30. Corrao G, Corazza GR, Bagnardi V. Mortality in patients with celiac disease and their relatives: A cohort study. Lancet 2001; 358: 356.

31. Holmes GK, Prior P, Lane MR Malignancy in celiac disease: Effect of a gluten free diet. Gut 1989; 30: 333.
32. AsKung J, LnNet M, Gridiey G. Cancer incidence in a population-based cohort of individuals hospitalized with celiac disease or dermatitis herpetiformis. Gastroenterology 2002; 123: 1428.

33. Cown P, Reunala T, Pukrala E. Celiac diseaseassociated disorders and survival. Gut 1994; 35: 1215.

34. Janatuinen EK, Kemppainen TA, Julkunen RJ, Kosma VM, MäKI M, HeIKKINen M ET AL. No harm from five-year ingestion of oats in celiac disease. Gut 2002; 50: 332.

35. Schmiтz J. Lack of oats toxicity in celiac disease: Toxic fraction makes up less of total protein than in other cereals (editorial). BMJ 1997; 314: 159.

36. Peraaho M, Kaukinen K, Mustalahti K. Effect of an oats containing gluten-free diet on symptoms and quality of life in celiac disease: a randomized study. Scand J Gastroenterol 2004; 39: 27-31.

37. StorsRud S, Olsson M. Adult celiac patients do tolerate large amounts of oats. Eur J Clin Nutr 2003; 57: 163-9.

38. Mckinley M, LeiBowitz S, Bronzo R. Appropriate response to pneumococcal vaccine in celiac sprue. J Clin Gastroenterol 1995; 20: 113.

39. LeE SK, Lo W, MeMEO L Duodenal histology in patients with celiac disease after treatment with a gluten-free diet. Gastrointest Endosc 2003; 57: 187.

40. Selby WS, Painter D, Cowns A. Persistent mucosal abnormalities in celiac disease are not related to the ingestion of trace amounts of gluten. Scand J Gastroenterol 1999; 34: 909.

41. Trier J, Falchuk Z, Carey M, Schreiber D. Celiac sprue and refractory sprue. Gastroenterology 1978; 75: 307-16.

42. Trier J. Celiac sprue. N Engl J Med 1991; 325: 1709-19.

43. BARRY R, READ A. Celiac disease and malignancy. QJM 1973; 42: 665-73.

44. Hamlion J, Chambers R, Wynn-Wimams A. Role of gluten, prednisone and azathioprine in nonresponsive celiac disease. Lancet 1976; 1: 121316.

45. Rolny P, Sigurjonsottir HA, Remotti H. Role of immunosuppressive therapy in refractory spruelike disease. Am J Gastroenterol 1999; 94: 219.

46. Vaidya A, Bolanos J, Berkelhammer C. Azathioprine in refractory sprue. Am J Gastroenterol 1999; 94: 1967.

47. Maurino E, Niveloni S, Chernavsky A. Azathioprine in refractory sprue: Results from a prospective, 
open-label study. Am J Gastroenterol 2002; 97: 2595.

48. LONGSTRETH GF. Successful treatment of refractory sprue with cyclosporine. Ann Intern Med 1993; 119: 1014.

49. Giwett H, Arnott I, Mc intyre M, Campbell S, Dahele A, Priest M et al. Successful Inßiximab Treatment for Steroid-Refractory Celiac Disease: A Case Report. Gastroenterology 2002; 122: 800-5.

50. Holmes GKT, Stokes PL, Sorahan TM, Prior P, Waterhouse JAH, Cooke WT. Celiac disease, gluten-free diet and malignancy. Gut 1976; 17: 612-19.

51. Howdie PD, Jalal PK, Holmes GKT, Houlston RS. Primary smallbowel malignancy in the UK and its association with celiac disease. Q J Med 2003; 96: 345-53.

52. Green PH, Fleischauer AT, Bhagat G. Risk of malignancy in patients with celiac disease. Am J Med 2003; 115: 191.

53. Askung J, Linet M, Gridiey G, Halstensen TS, Eкsтrom K, Еквом A. Cancer incidence in a population-based cohort of individuals hospitalized with celiac disease or dermatitis herpetiformis. Gastroenterology 2002; 123: 1428-35.

54. Farré C, Domingo-Domenech E, Font R, Marques T, De Sevilia AF, Alvaro T et al. Celiac disease and lymphoma risk: a multicentre case-control study in Spain. Dig Dis Sci 2004; 49: 408-12.

55. FREEMAN HJ. Lymphoproliferative and intestinal malignancies in 214 patients with biopsy-defined celiac disease. J Clin Gastroenterol 2004; 38: 42934.

56. CARD TR, West J, Holmes GKT. Risk of malignancy in diagnosed celiac disease: a 24-year prospective, population-based cohort study. Aliment Pharmacol Ther 2004; 20: 769-75.

57. West J, Logan RFA, HiL PG, Loyd A, Lewis S, HuBBARD R ET AL. Seroprevalence, correlates, and characteristics of undetected celiac disease in England. Gut 2003; 52: 960-5.

58. SNook JA, Dwyer L, LeE-Euott C, Khan S, Wheeler DW, Nicholas DS. Adult celiac disease and cigarette smoking. Gut 1996; 39: 60-2. 